\title{
Optimal Allocation of Electric Power Distributed Generation on Distributed Network Using Elephant Herding Optimization Technique
}

\author{
R. Vijay \\ Assoc. Professor, CVR College of Engineering/ EEE Department, Hyderabad, India \\ Email: vijai.mtp@cvr.ac.in
}

\begin{abstract}
This paper deals with optimal allocation of distributed generation in the electrical distribution system. Due to rapidly increasing energy demand on the distribution network, the system is experiencing disturbances like equipment overloading, voltage sags and swell. In this paper, the thermal and power loss constraints are considered for optimal operation. Elephant Herding Optimization (EHO) technique is applied for optimal placement and sizing of distributed generation on electric distribution network. The conventional optimization technique fails due to its complexity while solving the nonlinear problems. The EHO technique is tested on 5 bus radial distribution system. The intelligent and precise allocation of distributed generation in electric distribution network by using EHO reduces the overloading of the equipment, voltage swell \& sag, active power, reactive power and production cost of electricity. Furthermore, the suggested optimization technique is expanded to 24 bus radial distribution and practical Indian system.
\end{abstract}

Index Terms: distributed generation, optimal allocation, renewable energy sources, market liberalization, elephant herding optimization

\section{INTRODUCTION}

Recently the initiatives on smart grid and sustainable energy Distributed Generations (DG) are playing an important role in electric power systems. In order to overcome energy demand, the advantages of DG [1] are used to their potential. The future active network will effectively and efficiently link small and medium scale electric power sources with consumer demands. Due to rapidly increasing energy demand on the distribution network, the system is experiencing disturbances like overloading the equipment's, voltage sags \& swell, thermal constraints and power loss.

The current development in small-sized and modulated power generation technologies have led to large-scale deployment of distributed generation in electrical distribution network [2]. The optimal allocation of distributed generation may provide enormous techno economic and social benefits such as minimized power/energy loss. The optimal location of distributed generation [3] in the electric distribution network is a nonlinear optimization problem, generally comprised of number, location and sizes.

The optimal placement, size and location in the distributed generation have been developed and effectively employed using conventional methods $[4,5]$. These methods have experienced slow convergence rate in concerned search space. Both real as well as reactive power loss minimization are the objectives for the DG placement [6] by using analytical methods. The conventional analytical methods [7] are not appropriate for solving complex problems. A boundary-based algorithm is used to obtain distributed generation regulates the individual best by snubbing the consequence of other objectives [8]. The allocation of distributed generation in electrical distributed network problem becomes a multi objective optimization [9] problem in which two or more objectives are optimized. The objective is inconsistent, so multiple objectives are converted into a single-objective problem by assigning weights.

Meta-heuristic and heuristic methods suggest the new practicable and streamlined elucidation. The Heuristic optimization algorithm is introduced to solve the optimal allocation of electrical distributed generation on distributed networks. Genetic algorithm [10] is used to abide generation, so that loss expenses and network disruption are minimized. Meanwhile the rating of the generator becomes exploited. The various optimization techniques such as Tabu Search (TS) [11], Particle swarm Optimization (PSO) [12], Artificial Bee Colony (ABC) [13], Bat Motivated Optimization (BMO) [14], Ant Colony Optimization (ACO) [15], Bacterial Swarm Optimization (BSO) [16,17], Enriched Biogeography Based Optimization (EBBO) Algorithm [18] are introduced to elucidate the optimal allocation in distribution network successfully. The hybrid optimization algorithm is comprised of Improved MultiObjective Harmony Search (IMOHS) [19] and is applied to assess the sway of DG placement for an optimal arrangement of a distribution system.

The above revealed heuristic approach is used to determine the optimal location and sizing of DG from an investment point of view. In these literatures, the Short Circuit Bound (SCB) restrictions are not deliberated. The emphasis of the objective function is to optimize cost preferably than increasing renewable energy resources. In this paper this SCB and capital investment in new renewable distributed generation units are considered for optimal operation.

A new simple nature inspired optimization technique called the Elephant Herding Optimization (EHO) [20] algorithm stimulated by the elephant herding behavior is used. The EHO method is inspired by the herding behavior of male and matriarch (female) elephant. The food and shelter searching technique is the foremost inspirations in this algorithm. This EHO method is smeared to solve the optimal placement of distributed generation on electrical 
distributed network. In this paper, 5 bus radial distribution networks are deliberated and unraveled for optimal site in the distribution network.

The fragmentations of this paper are alienated into five sections, Section 2 explains about the optimal placement of distributed generation problem formulation. Section 3 presents the overview of the proposed EHO algorithm. Section 4 discusses the application of EHO for optimal placement of distributed generation. Section 5 deals with detailed results and discussion followed by conclusion in Section 6.

\section{Problem Formulation}

Embedded Generation is the small capacity generation and is not associated to the transmission system. Recently more amount of generation is being coupled at distribution level. This leads to change the features of the power system network. To increase the amount of power generation, change in the planning and design of the electrical distribution network is prerequisite.

Most of embedded generation is from renewable energy sources. The optimal usage of the prevailing power system network leads to cost operative method. Electric power generation capability should be apportioned across the buses, allowing for all the practical restrictions. The electric power generation capacity should be maximized [3]. Hence the proposed impartial function is taken as

$$
J=\sum_{n=1}^{N} P_{D G n}
$$

Where $P_{D G n}$ is the DG power generation capability at the $n^{\text {th }}$ bus and without loss, presumed that there is a generator fulfilling every bus. The impartial function $J$ (MW) is maximized subject to the restrictions.

\section{A.Thermal Constraint:}

The rated $I$ of the transmission lines need not to be surpassed

$$
I_{n}<I_{n}^{\text {rated }}, n \forall N
$$

Where $I$ is the current circulated from the generator to bus and $I^{\text {rated }}$ is the maximum line current.

\section{B.Equipment Ratings:}

The following ratings considered here are transformer capacity, short circuit bound, short circuit proportion and voltage rise outcome, the detailed explanations as follows

\section{Transformer Capacity:}

The amount of power generation associated minus varying peak seasonal (summer) load didn't surpass the higher voltage evaluation of the transformer.

$$
P_{T r} \leq P_{T r C a p}
$$

Where $P_{T r}$ indicates the power flow through substation transformer and $P_{T r C a p}$ directs the rating of transformer taken.

\section{Short Circuit Bound:}

The short circuit ratings are calculated and mentioned in the distribution code. The SCB calculation is derived to fortify that this constraint has not transcended the installed capacity. The restriction is specified by

$$
S C B_{T X}<S C B_{\text {Rated }}
$$

$S C B_{\text {rated }}$ is the maximum current that breaks the switchgear securely in the abnormal condition. The endowment of power generation at each bus to SCB is resolved by short circuit analysis. The SCB endowment of power generation at each bus are consolidated into an algebraic equation as follows

$$
\sum_{m=1}^{N} \zeta_{m T x} P_{D G m}+\sigma T_{x} \leq S C B_{\text {rated }}
$$

Where $\zeta_{m T x}$ is the dependence of the SCB at the transmission substation to power inoculation at bus $m$ and $\sigma T_{x}$ is the inceptive SCB of the transmission bus.

Short Circuit Proportion (SCP):

The SCP is defined as a proportion of the power generated $P_{D G}(\mathrm{MW})$ at every individual bus to the Short Circuit Level at every individual bus. The voltage dip near the generator due to outage of feeder is indicated. When induction motors are placed in the circuit it leads to voltage instability.

$$
\frac{P_{D G n}}{S C B_{n} \cdot \cos (\varphi)} * 100 \leq 10 \%, \not \forall N
$$

Where $S C B_{n}$ refers to the SCB at the $n^{\text {th }}$ bus and $\cos (\phi)$ is the power factor of the generator. The base value for the SCB at $n^{\text {th }}$ bus is calculated with no power generation. The SCB at the $n^{\text {th }}$ bus is

$$
\begin{aligned}
& S C B_{n}=\sigma_{n}+\sum_{m=1}^{N} \zeta_{m n} P_{D G m} i \neq j, n \forall N \\
& P_{D G n}-0.1 \cos (\varphi) \sum_{m=1}^{N} \zeta_{m n} P_{D G m} \leq 0.1 \cos (\varphi) * \delta_{i}
\end{aligned}
$$

Voltage Rise Outcome (VRO):

The voltage at the generator is specified by below mentioned eqn.

$$
V_{G}=V_{L}+\frac{R P_{L}+X Q_{L}}{V_{L}}+j \frac{X P_{L}-R Q_{l}}{V_{L}}
$$

$P_{L}$ and $Q_{L}$ are active and reactive power at the bus, $V_{G}$ is the voltage at the generator and $V_{L}$ is the voltage at the respective bus. Thus, it is comprehended that the generator voltage is the bus or load voltage and the rate associated with line impedance and power flow through the transmission line.

The active power flow through the electric distribution system has a large influence on voltage for the reason that the distributed network is high resistance compared to other 
transmission lines. This steers to approximately higher $\mathrm{X} / \mathrm{R}$ value compared to transmission networks. The system voltage of each bus must lie within standard limits.

$$
V_{n}^{\min } \leq V_{n} \leq V_{n}^{\max }
$$

Where $V_{n}^{\min }$ and $V_{n}^{\max }$ indicates the minimum and maximum voltage boundaries at the $n^{\text {th }}$ bus. The attributes of voltage and power inoculations at every bus is resolute.

$$
\mu_{n} P_{D G n}+\beta_{n}+\sum_{m=1}^{N} \mu_{m n} P_{D G n} \leq V_{n}^{\max } n \forall N
$$

Here $\mu_{i}$ is the dominion of the voltage level at bus $n$ taking place on power inoculations at bus $n$. That is the slope of the generated voltage vs power inoculation attributes of the $n^{\text {th }}$ bus. $\beta_{n}$ mentions the inceptive voltage level at the $n^{\text {th }}$ bus with no generation and $\mu_{n m}$ refers to the reliance of the voltage level at bus on power injections at bus.

This dissection is accomplished under minimum load conditions as it is the worst condition for voltage rise.

\section{ELEPHANT HERDING OPTIMIZATION}

The EHO [21] search method is based on the steering elephant clans. In existing biosphere, the elephants associated with dissimilar clans stay organized under the directorship of female elephant; while the males when they grow leaves their elephant family groups.

The elephant behavior in search of food and shelter is modeled in two ways. These include clan apprising machinist and unraveling machinist. The current position of every group is updated by the matriarch, this process is known as clan apprising machinist. The enactment of the clan unraveling machinist enhances the elephant population miscellany in the whole search space.

Elephant herding optimization is implemented by the following procedure

- The elephant population is tranquil in some clans and every fraternity has fixed numbers.

- The male elephants move away from their clan and live distant from their clan at every iteration.

- The elephants in every group stay organized beneath the directorship of matriarch.

\section{Clan updating operator:}

The elephant location in clan $C_{n}$ is effected by matriarch $C_{n}$ in all iterations. The elephant $m$ in $\operatorname{clan} C_{n}$, is restructured by the following equation

$$
e_{n e w, c n, m}=e_{c n, m}+\lambda \cdot\left(e_{b e s t, c n}-e_{c n, m}\right) \cdot r
$$

From the above equation $e_{n e w, c n, m}$ is recently rationalized location of elephant $m$ in clan $C_{n}$ and $e_{c n, m}$ is the old location of elephant $m$ in group $C_{n}$ respectively. $\lambda$ is the surmounting factor that regulates the impact of matriarch
$C_{n}$ on $e_{c n, m}$ and the assessment of $\lambda$ lies between $[0,1]$. $e_{\text {best,cn }}$ represents the female elephant $C_{n}$ which is the fittest distinct elephant in clan $C_{n}, r$ represents the stochastic and uniform distribution in the assortment [0,1]. The fittest elephant in each clan is updated by eqn.(12), i.e. $e_{c n, m}=e_{b e s t, c n}$. If the global optimal solution is not satisfied then again compute eqn. (13)

The fittest elephant is again rationalized by the below equation

$$
e_{\text {new }, c n, m}=\kappa \cdot e_{\text {center, } c n}
$$

Where $\kappa$ governs the effect of the $e_{\text {center,cn }}$ on $e_{\text {center,cnm }}$. The new position $e_{\text {new }, \text { cn }, m}$ shown in eqn. (13) is determined from the information of the elephants in clan $C_{n} \cdot e_{\text {center,cn }}$ is the center of elephant group $C_{n}$ and for the $x^{\text {th }}$ dimension it is premeditated by

$$
e_{\text {center }, \mathrm{cn}, \mathrm{x}}=\frac{1}{n} * \sum_{m=1}^{N} e_{c n, m, x}
$$

Where $1 \leq x \leq X$ indicates the $x^{\text {th }}$ dimension and $X$ is its total dimension. $N$ is the number of elephant population in group $C_{n} \cdot e_{c n, m, x}$ is the $x^{\text {th }}$ dimension of the individual elephant. The Centre of clan $C_{n}, e_{c e n t e r, c n}$ is calculated by eqn.(14).

\section{Clan Separating Operator:}

In the elephant's clan male elephants move away from their family group and live solitary. This process is modeled as clan separating \& updating operator, which is used for cracking optimizing hitches. The poorest fitness elephant is eliminated from the clan at each iteration, so that the search ability of EHO method is improved. This worst fitness elephant group is calculated by

$$
e_{\text {worst }, c n}=e_{\min }+\left(e_{\max }-e_{\min }+1\right) * r
$$

Where $e_{\max }$ and $e_{\min }$ are maximum bound and minimum bound of each individual location. $e_{\text {worst,cn }}$ is the poorest individual location in the clan $C_{n} . r \in[0,1]$ is the stochastic and uniform distribution in the range $[0,1]$.

\section{Pseudo Code - Clan Updating Machinist:}

For

\{

$C_{n}=1$ to $N_{\text {clan }}$ (all groups in elephant population) do

For

\{

$m=1$ to $N_{c n}$ (for entire elephant in group $C_{i}$ ) do 


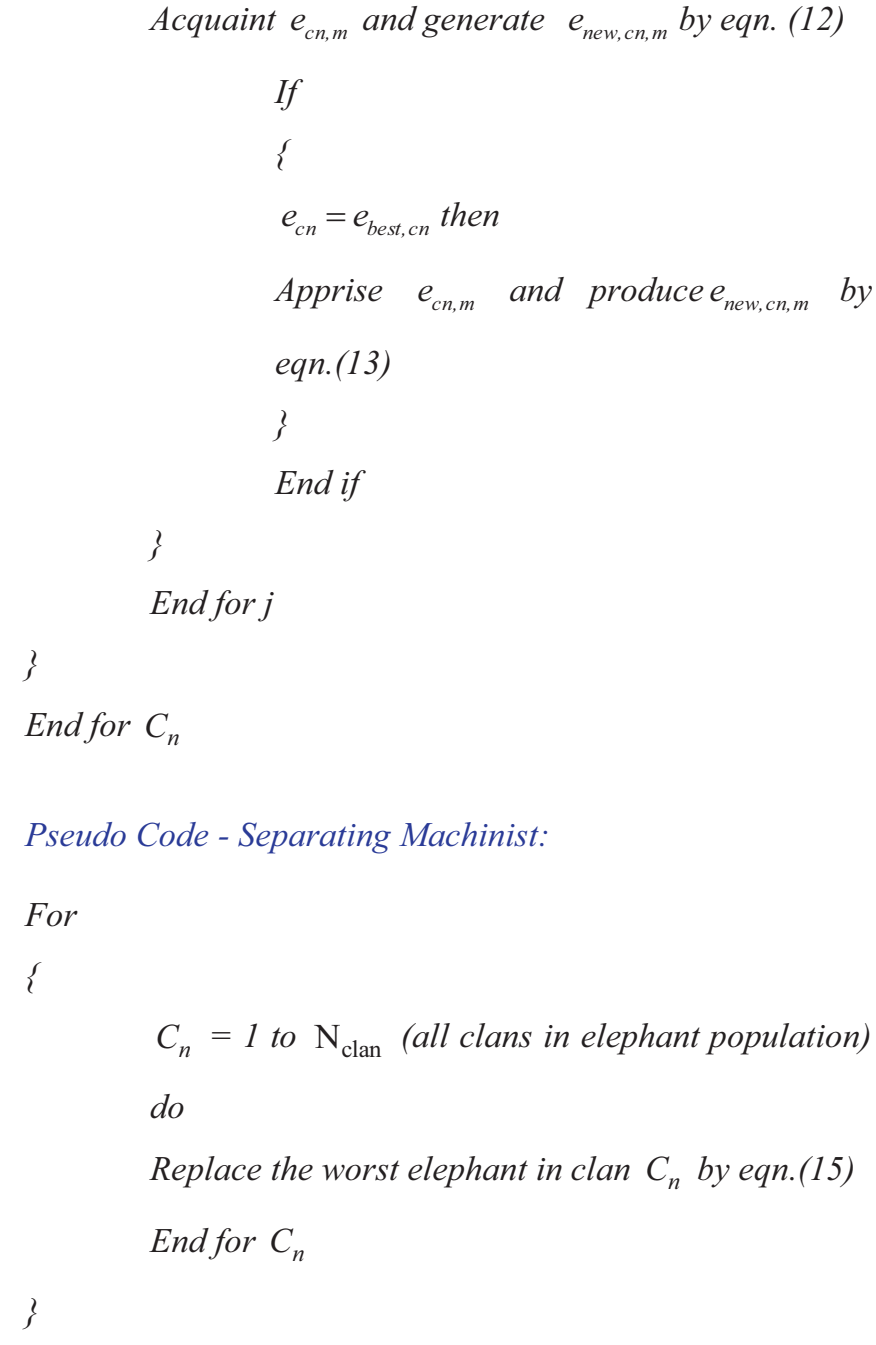

Pseudo Code - EHO Algorithm:

Initialize the population; Fix generation countert=1;

\section{IMPLEMENTATION OF EHO FOR OPTIMAL ALLOCATION OF DISTRIBUTED GENERATION ON DISTRIBUTION NETWORK}

Usually elephants form some groups and move in search for food and shelter. In this context, the elephant movement from one location to another location is considered as power flow from one bus to another bus. They update their position and location status. Correspondingly, the optimal allocation problem is related to the bus location and bus data.

Elephants communicate with each other by low frequency vibrations. When they find the worst case of disturbances in the search space, in a particular place, they communicate with each other's in the clan (groups). Meanwhile, low voltage and high voltage fluctuations are the main cause of disturbance and losses in the distribution network. In a distribution network due to unexpected change in load, the bus voltage starts fluctuating. Depending on the voltage fluctuations, the weaker buses are identified.

The best position treasure by the elephant is the best bus (voltage profile) location. The position of the elephant location with more disturbances is the best position for placement of distributed generation.

The following steps explain the implementation of EHO for optimal DG allocation in distributed networks

Step 1: Set max number of elephants, no of elephants, no of buses, bus data node voltages, short circuit bound, $\lambda$ and $\kappa$.

Step 2: Update the best and worst position of the elephant, i.e., best and worst bus voltage.

Step 3: Update the worst position of elephant i.e., worst bus voltage.

Step 4: Elephants will communicate with others to update the current worst position (Local Solution) among the iteration.

Step 5: Now the elephants will keep on herding to find the global best position (Worst Location). By discovering the worst position, the voltage profile is enriched by employing distributed generations in the weak bus.

\section{Maximum Generation MaxGen}

While

\{

t, Max Gen do

Sort all the elephants according to their fitness.

Implement clan updating operator

Implement separating operator as shown

Evaluate population by the newly updated positions

$$
T=t+1
$$

End while

\section{\}}




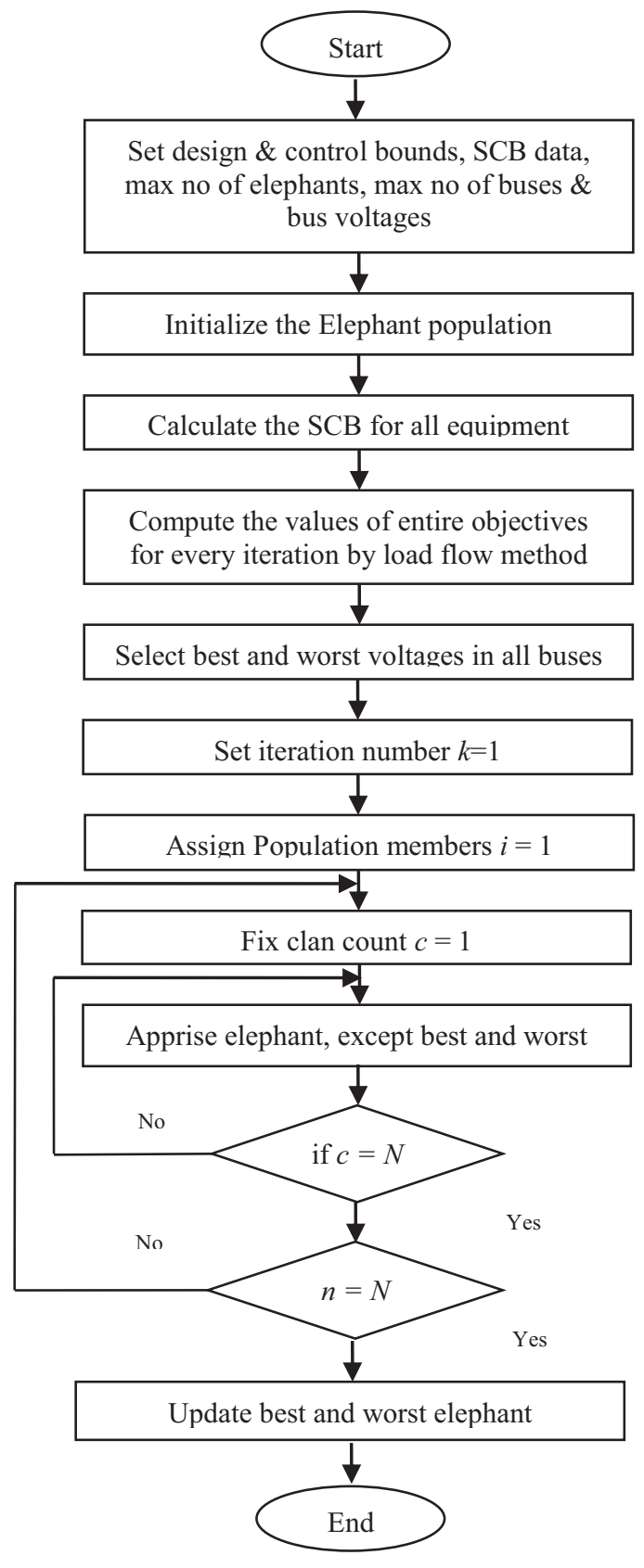

Figure 1. Flowchart of Implementing EHO to optimal placement of distributed generation

\section{RESUlts AND DisCUSSION}

The elephant herding optimization technique is tested on 5 bus radial distribution systems [3]. The tail fed 38/110 kV station with 5 buses is shown in Fig. 2. The EHO is verified to test on practical heftier networks. This segment discusses the result illustrations and demonstrates the potential for network sterilization.

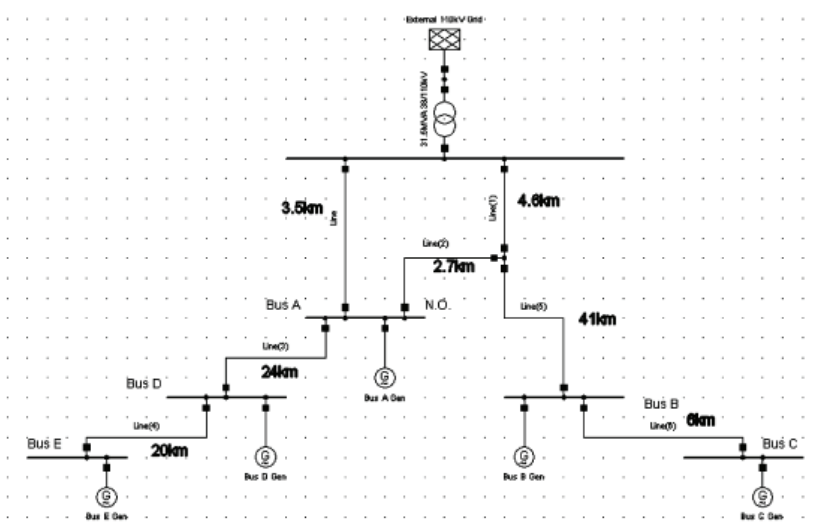

Figure 2. 38 KV 5 Bus Radial Distribution Network Diagram

By injecting power at various buses, the solitary voltage sensitive attributes are formed and presented in Fig.3.

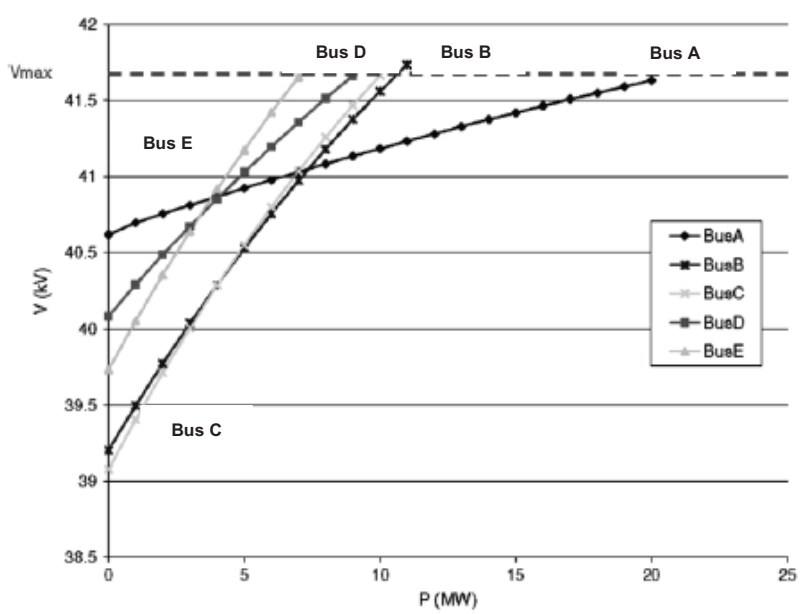

Figure 3. Generated Power Injections at respective Buses and its Voltage Compassions

By using the solitary voltage sensitive attributes, it is found that bus $\mathrm{A}$ is slight profound to power instillations at different buses. The bus $\mathrm{A}$ is slightly profound due to the location which is very near to $38 / 110 \mathrm{kV}$ station. The bus B \& $C$ have analogous characteristics because those buses connected separately as of the distribution network.

TABLE I.

VOLTAGE INTERDEPENDENCIES OF 5 BUS SYSTEM

\begin{tabular}{|c|c|c|c|c|c|}
\hline$\mu$ & $\mathbf{A}^{\text {th }} \mathbf{B u s}$ & $\mathbf{B}^{\text {th }} \mathbf{B u s}$ & $\mathbf{C}^{\text {th }} \mathbf{B u s}$ & $\mathbf{D}^{\text {th }} \mathbf{B u s}$ & $\mathbf{E}^{\text {th }} \mathbf{B u s}$ \\
\hline $\mathbf{A}^{\text {th }} \mathbf{B u s}$ & 0.053 & 0.008 & 0.007 & 0.021 & 0.016 \\
\hline $\mathbf{B}^{\text {th }}$ Bus & 0.012 & 0.218 & 0.18 & 0.009 & 0.007 \\
\hline $\mathbf{C}^{\text {th }}$ Bus & 0.012 & 0.191 & 0.238 & 0.009 & 0.007 \\
\hline $\mathbf{D}^{\text {th }}$ Bus & 0.026 & 0.008 & 0.007 & 0.162 & 0.11 \\
\hline $\mathbf{E}^{\text {th }}$ Bus & 0.026 & 0.008 & 0.007 & 0.135 & 0.234 \\
\hline
\end{tabular}

The values of $\mu_{n}(k V / M W)$ are determined from Fig.3. Where $\mu_{n}$ is the reliance of voltage in the bus $n$ on the power inoculation at bus $n$. The gradient voltage vs power 
inoculation attributes of $n^{\text {th }}$ bus, the values of $\mu_{n}$ are presented diagonally in Table I.

The values of $\mu_{n m}$ are presented in Table I as off diagonal elements. Where $\mu_{n m}$ is the voltage reliance at bus $n$ on the power inoculation at bus $m$.

The individual sensitivity of SCB at $38 / 110 \mathrm{kV}$ substation to power injection at distinct bus is premeditated and presented in Fig.4.

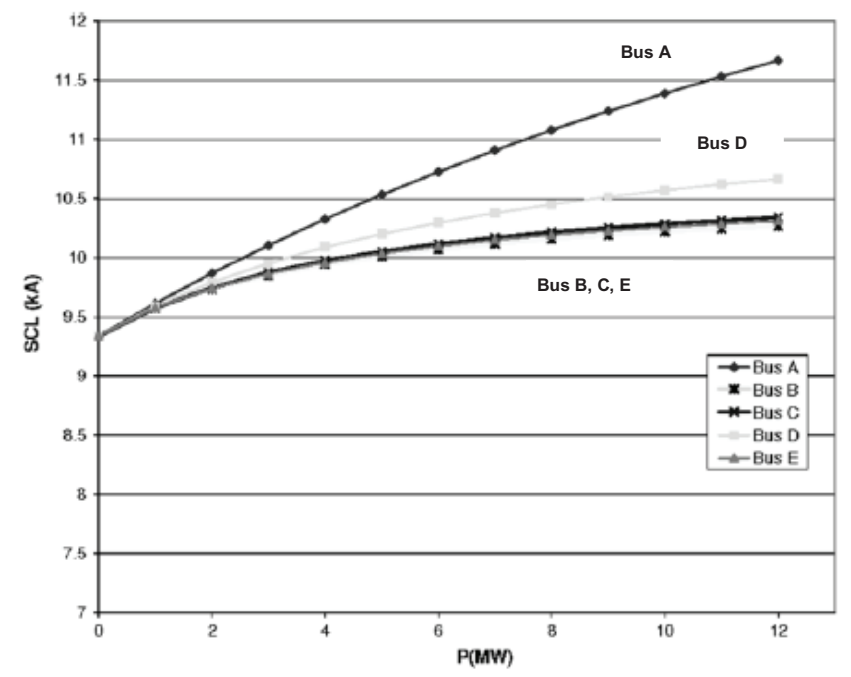

Figure 4. SCB at $38 / 110 \mathrm{kV}$ substation vs. Power Injections at Individual Buses

The effect of bus on the SCB at $38 / 110 \mathrm{kV}$ substation is reliant on the remoteness from the substation. The influence of SCB is very high to bus A as the position of bus A is close to $110 \mathrm{kV}$ substation.

TABLE II.

$S C B_{T x}$ Dependencies of 5 Bus Systems

\begin{tabular}{|c|c|c|c|c|c|}
\hline Dependency Factor/ Bus & Bus A & Bus B & Bus C & Bus D & Bus E \\
\hline$\zeta_{T x}(\mathrm{kA} / \mathbf{M W})$ & 0.18 & 0.11 & 0.10 & 0.14 & 0.11 \\
\hline
\end{tabular}

The values of $\zeta_{n T X}(\mathrm{kA} / \mathrm{MW})$ are revealed in Table II. Where $\zeta_{n T X}$ is SCB reliance at the substation to power inoculation at bus $m$. The slope of SCB vs. power inoculation attributes of the $m^{\text {th }}$ bus is revealed in Fig.4.

\section{Case 1: Consumer power generation on $E^{\text {th }}$ bus}

In this case, 7MW of consumer power generation is apportioned to bus E. Using EHO algorithm, sensitivity values of the maximum apportionment are indomitable, after 20 epochs the power generation inclines to $10.6 \mathrm{MW}$ and is exposed in Table III.

\section{Case 2: Without preallocated generation}

In this case no generation is prior apportioned. The EHO is smeared and the whole allocation after 20 epochs is resolute as $20.65 \mathrm{MW}$.

TABLE III.

Optimal Allocation of DG in DifFERENT CASES CONSIDERED

\begin{tabular}{|c|c|c|c|c|}
\hline \multirow[b]{3}{*}{ Bus No } & \multicolumn{2}{|c|}{$\begin{array}{c}\text { Linear Programming } \\
\text { Algorithm [3] }\end{array}$} & \multicolumn{2}{|c|}{ EHO (Proposed) } \\
\hline & Case 1 & Case 2 & Case 1 & Case 2 \\
\hline & $\begin{array}{c}\text { Actual } \\
\text { Power in } \\
\text { bus } \\
\text { (MW) }\end{array}$ & $\begin{array}{c}\text { Power } \\
\text { Injected } \\
(\mathrm{MW})\end{array}$ & $\begin{array}{c}\text { Power } \\
\text { Injected } \\
\text { (MW) }\end{array}$ & $\begin{array}{l}\text { Power } \\
\text { Injected } \\
(\mathrm{MW})\end{array}$ \\
\hline$A^{\text {th }}$ Bus & 0 & 4.06 & 0 & 3.95 \\
\hline B $^{\text {th }}$ Bus & 0 & 4.30 & 0 & 3.82 \\
\hline $\mathrm{C}^{\text {th }}$ Bus & 3.60 & 5.95 & 3.60 & 5.27 \\
\hline$D^{\text {th }}$ Bus & 0 & 5.31 & 0 & 4.86 \\
\hline $\mathbf{E}^{\text {th }}$ Bus* & 7.60 & 3.12 & 7.00 & 2.85 \\
\hline $\begin{array}{l}\text { Total Power } \\
\text { Injected }\end{array}$ & 11.20 & 22.74 & 10.60 & 20.65 \\
\hline
\end{tabular}

The EHO technique works on the radially functioned distribution system. The intensity of system decontamination diverges mutually between the buses. The distributed system structure is demonstrated in this literature with high level of dependency. In standby feeding circumstances entire five buses are associated to the transmission bus through the single bus. In a loftier power network, many numbers of transmission lines are connecting buses to the transmission bus with typical open points unscrambling sectors. This lessens the whole interdependence in the voltage levels, nevertheless none of SCB. Therefore, the possibility for power system decontamination is concentrated.

From Table III, it is clear that the proposed EHO technique outperforms the Linear Programming Algorithm by the means of the power injection considered in case 2. By using the EHO technique the power injected in the bus $\mathrm{E}$ and with respect to all other buses is also diminished comparatively, which shows the superiority of the proposed optimization technique. By using this EHO technique 9.19\% power injected is reduced, which is distinct from the Table III. Meanwhile, the charge incurred in the particular DG will be saved.

The EHO determines the optimal allocation from the capital asset and helps the distribution companies to identify the possibility of faults. The projected technique also reduces the dependency of the individual bus. Moreover, the DG allocation at the particular bus is optimized and improves the stability of the system.

\section{CONCLUSIONS}

This paper proposes the elephant herding optimization technique for optimal allocation and sizing of distributed generation on electrical distribution networks. DG is the impeccable elucidation of recent and time ahead challenges 
of modern electric power generation and distribution system. This might encounter the challenging desires of the consumers parsimoniously and ecologically by curtailing the charge, intricacy allied with on-site power generation, transmission and distribution. The proposed technique is applied on 5 bus radial power distribution system and the outcomes are presented. The result clearly indicates that the proposed EHO algorithm gives the optimal results. The proposed EHO technique is capable of producing higher quality results in terms of optimal allocation and sizing of distributed generation. The proposed EHO technique outperforms the conventional technique and the power injected by the DG in the weaker bus is also reduced considerably. The proper allocation and sizing of DG reduces the fuel cost to the distribution companies. Furthermore, this considered optimization technique may apply to the impending maneuver of the Indian Power distribution network for the proper constituting electric system.

\section{REFERENCES}

[1] El-Khattam and Walid, "Optimal investment planning for distributed generation in a competitive electricity market," IEEE Transactions on power systems, vol. 19, no. 3, pp. 1674$1684,2004$.

[2] G.P.Harrison and A. R. Wallace, "Optimal power flow evaluation of distribution network capacity for the connection of distributed generation," IEEE Proceedings Generation, Transmission and Distribution, vol. 152, no. 1, pp. 115-122, 2004.

[3] K.Andrew and Mark O'Malley, "Optimal allocation of embedded generation on distribution networks," IEEE Transactions on Power Systems, vol. 20, no. 3, pp. 16401646, 2005.

[4] S.K.Pandey, S.R.Mohanty and N.Kishor, "A literature survey on load-frequency control for conventional and distribution generation power systems," Renewable and Sustainable Energy Reviews, vol. 25, pp. 318-334. 2013

[5] R.Vijay and C.S. Ravichandran. "A detailed investigation on conventional and meta-heuristic optimization algorithms for economic power scheduling problems," International Journal of Engineering Trends and Applications, vol. 3, no. 4, pp. 4053, 2016.

[6] P.S. Georgilakis and N.D. Hatziargyriou, "Optimal distributed generation placement in power distribution networks:models, methods, and future research," IEEE Transactions on Power Systems, vol. 28, no. 3, pp. 3420-3428. 2013

[7] Paliwal.Priyanka, N. P. Patidar, and R. K. Nema, "A comprehensive survey of optimization techniques used for distributed generator siting and sizing," Southeastcon, Proceedings of IEEE. 2012.

[8] A. Zangeneh, S. Jadid, and A. Rahimi-Kian, "Normal boundary intersection and benefit cost ratio for distributed generation planning," Int. Trans.Elect. Energy Syst., vol. 20, no. 2, pp. 97-113, 2010.

[9] S. N. Naik, D. K. Khatod, and M. P. Sharma, "Analytical approach for optimal siting and sizing of distributed generation in radial distribution networks," IET Generation, Transmission, Distribution., vol. 9, no. 3, pp. 209-20, 2014.

[10] M. H. Moradi and M. Abedini, "A combination of genetic algorithm and particle swarm optimization for optimal DG location and sizing in distribution systems," Int. J. Elect. Power Energy Syst., vol. 34, no. 1, pp. 66-74, Jan. 2012
[11] M.Gandomkar, M.Vakilian and M.Ehsan, "A genetic based tabu search algorithm for optimal DG allocation in distribution networks. Electric Power Components and Systems, vol. 33, no. 12, pp. 1351-1362, 2005.

[12] M.Nazari-Heris, S. Madadi, M.P.Hajiabbas and B.Mohammadi-Ivatloo, "Optimal distributed generation allocation using quantum inspired particle swarm optimization," In Quantum Computing: An Environment for Intelligent Large Scale Real Application .pp. 419-432, 2018.

[13] R.Vijay and R.Sowmya, "Optimal sitting of PV-wind-energy storage system integrated micro grid using artificial bee colony optimization technique," International Journal of Innovative Research in Computer and Communication Engineering, Vol. 80, 2017.

[14] R.Vijay and C.S. Ravichandran, "Optimal placement and sizing of distributed power sources in micro grid for power loss minimization using bat motivated optimization algorithm" Asian Journal of Research in Social Sciences and Humanities, Vol. 6, No.8, pp. 252-266, 2017.

[15] R.Vijay and J. Durga Devi. "Optimal placement and sizing of wind-PV integrated power generation by ant colony optimization technique," International Journal of Recent Trends in Engineering \& Research, vol. 03, no. 5, pp. 24551457, 2017.

[16] R.Vijay, "Quorum sensing driven bacterial swarm optimization to solve practical dynamic power ecological emission economic dispatch," International Journal of Computational Methods, vol. 15, no. 03, pp.1850089-24,2018.

[17] R.Vijay and C.S. Ravichandran, "Scheduling practical generating system using an improved bacterial swarm optimization," Technical Gazette, vol.23, no. 5, pp. 1307$1315,2016$.

[18] V.Raviprabakaran "Optimal and stable operation of microgrid using enriched biogeography based optimization algorithm," Journal of Electrical Engineering, vol. 17, no. 4, pp. 1-11, 2018.

[19] K.Nekooei, M.M. Farsangi, H. Nezamabadi-Pour and K.Y.Lee, "An improved multi-objective harmony search for optimal placement of dgs in distribution systems," IEEE Transactions on smart grid, vol. 4, no. 1, pp. 557-567, 2013.

[20] Wang, Gai-Ge, Suash Deb and Leandro dos S. Coelho. "Elephant herding optimization," Computational and Business Intelligence (ISCBI), 3rd International Symposium on IEEE, 2015.

[21] N.K.Meena, S.Parashar, A.Swarnkar, N.Gupta and K.R.Niazi, "Improved elephant herding optimization for multiobjective DER accommodation in distribution systems", IEEE Transactions on Industrial Informatics, vol. 14, no. 3, pp.1029-1039, 2018. 